\title{
8
}

\section{Reservoir Operation Optimization by Reinforcement Learning}

\author{
M. Mahootchi, H. R.Tizhoosh, and K. Ponnambalam
}

Planning of reservoir management and optimal operations of surface water resources has always been a critical and strategic concern of all governments. Today, many equipments, facilities, and substantial budgets have been assigned to carry out an optimal scheduling of water and energy resources over long or short periods. Many researchers have been working on these areas to improve the performance of such a system. They usually attempt to apply new mathematical and heuristic techniques to tackle a wide variety of complexities in real-world applications and especially large-scale problems. Stochasticity, nonlinearity/nonconvexity and dimensionality are the main sources of complexity. In other words, there are many techniques, which could circumvent these complexities via some kind of approximations in uncertain environments with complex and unknown relations between various system parameters. In fact, using different methods to optimize the operations of large-scale problems coming along with much unrealistic estimations makes the final solution very imprecise and usually too far from real optimal solution. Moreover, the existing limitations of hardware or software cause some important physical constraints, which prevent various relations between variables and parameters from being considered. In other words, even if all possible relations between parameters in a problem are known and definable, considering all of them simultaneously might make the problem very difficult to solve.

Mahootchi, M., H.R. Tizhoosh and K. Ponnambalam. 2007. "Reservoir Operation Optimization by Reinforcement Learning." Journal of Water Management Modeling R227-08. doi: 10.14796/JWMM.R227-08. (C) CHI 2007 www.chijournal.org ISSN: 2292-6062 (Formerly in Contemporary Modeling of Urban Water Systems. ISBN: 0-9736716-3-7) 
In an optimization model of a real-world application of reservoir operations, there usually exist different objective functions and numerous linear and non-linear constraints. Thus, if the number of variables and parameters in this model make the problem intractable and too large, existing software or hardware might not be able to find an optimal solution using conventional optimization methods in a reasonable time. For example, stochastic dynamic programming (SDP), a well-known technique in the reservoir management, suffers seriously from the curse of dimensionality and of modeling in multi-reservoir systems. Therefore, to overcome this challenge, several ideas have been developed and implemented in past decades: dynamic programming successive approximations (DPSA) (Larson, 1968), incremental dynamic programming (IDP) (Hall et al., 1969), multilevel incremental dynamic programming (MIDP) (Nopmongcol \& Askew, 1976), and different aggregation decomposition methods (Turgeon, 1981; Ponnambalam, 1987; Ponnambalam \& Adams, 1996).

Using simulation along with optimization techniques could be a promising alternative in water resources management. Labadie believes that a direct linkage between simulation and implementation of reservoir optimization algorithms could be an important key to success in reservoir management in the future (Labadie, 2004).

Different reinforcement learning (RL) techniques, as simulation-based optimization techniques, might be suitable approaches to overcome the curse of dimensionality or at least decreases this difficulty in real-world applications. The mechanism of learning in these approaches is based on interacting with an environment and receiving immediate or delayed feedback through taking actions (Watkins \& Dayan, 1992, Sutton \& Barto, 1998). In other words, these techniques could start learning without a priorknowledge of the stochastic behavior of the system; therefore, they are called model-free methods. This means that they do not need to know anything about the behavior of the system at the starting point of the learning process. The agent or decision-maker begins from an arbitrary situation and attempts to interact with the environment. During these interactions, the agent experiences new situations, saves the results and uses them in the future decision-making. It is clear that in the beginning of the learning, for most of the time, the agent encounters new situations which have never been observed. In this situation, the action taken is not based on a prior knowledge. However, after having enough interactions with the environment, the agent can slowly understand the behavior of the system, and thereafter it attempts to utilize this knowledge for more accurate 
decision-making. Furthermore, the agent usually looks for finding new information about the environment by taking an action randomly.

In fact, different techniques in RL are able to learn continually. In other words, they could be applied in on-line (real time) or off-line (simulation) learning. Using RL in on-line learning from scratch (without any prior knowledge) could be very expensive and troublesome; therefore, it could be initially used as an off-line learning during which a basic understanding of the environment is achieved. This knowledge could be eventually useful to start an on-line learning.

In most real-world applications, the dynamic of the system is continuously changing. RL techniques are substantially able to adapt itself to these changes and to generate adequate responses and reactions to them. Furthermore, in some optimization techniques such as SDP, the final policy should cover all possible states in the system while many of them are practically impossible or unimportant. However, In RL, because of using simulation or on-line interaction with the environment, the focus is on the significant states or those states, which are practically possible.

In this study, one of the well-known and popular techniques in RL called Q-Learning is used to find an optimal closed-loop operational policy in a single-reservoir problem with linear objective functions, considering the stochastic nature of inflows into a reservoir. This could be a starting point to tackle the difficulty of finding an optimal solution for multi-reservoir applications in the future. Like the SDP method, the release from the reservoir is a decision variable that should be determined for every storage level as a system state, that is, the water stored in the reservoir. It is assumed that inflow into the reservoir is a normally distributed random variable. Two types of creating admissible actions including the optimistic and pessimistic schemes are investigated. Based on preliminary results in the simulation, the performance of the Q-Learning method will be measured and compared with the results of the SDP technique.

\subsection{Reinforcement Approach to Reservoir Management}

There are four main components in any RL system: policy, reward, value function, and model. Of course, a model is optional and there is no necessity to be included in RL (Sutton \& Barto, 1998). The policy is a mapping from state to decision to be made. The reward is the immediate response of 
environment to the action taken by the agent. The action-value function, which is defined for an action-state pair, takes into account the cumulative reward from starting point of RL. In other words, the value function, in contrary to reward function, specifies the gain of the system in a given pair of action-state after a long run. This is actually a basis for taking action in different situations by the agent. The model component of RL determines the next state and reward of the environment based on a mathematical function. This is actually necessary when the process of learning is going to be performed off-line. It means that RL is going to be implemented based on simulation using the specified model to determine the response of the respective environment to the action taken in the current state. The process of learning is illustrated schematically in Figure 8.1.

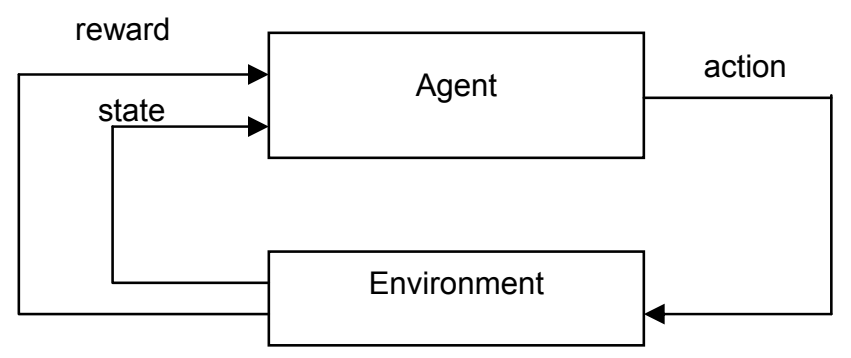

Figure 8.1 The schematic view of reinforcement learning.

\subsubsection{Action-value Function}

As mentioned before, the criterion, which the agent has to consider for taking an action, is the accumulated rewards or value functions. The value function here has the same meaning in the conventional method of dynamic programming with two main parts: immediate reward and accumulated reward. Because in RL the learning process is implemented by a direct interaction with the environment, value functions have to be updated after each interaction. This is similar to the asynchronous dynamic programming in which the latest updated value functions of states can be used for updating value functions of other states in the same iteration (Gosavi, 2003). We can easily extract the formulation of updating value functions in RL from SDP.

The value function formula in the SDP method is as follows: 


$$
\begin{gathered}
V_{t}^{(k)}(i)=\max _{a \in \mathrm{A}(i, t)}\left[\sum_{j=1}^{N} P_{t}^{a}(i j) \times\left(f_{t}(i, a)+\gamma V_{t+1}{ }^{(k-1)}(j)\right)\right] \\
\sum_{j=1}^{N} P_{t}(i, a, j) \times f_{t}(i, a)=\mathrm{Re} \\
V_{t}^{(k)}(i)=\max _{a \in \mathrm{A}(i, t)}\left(\mathrm{Re}+\gamma \sum_{j=1}^{N} P_{t}^{a}(i j) V_{t+1}{ }^{(k-1)}(j)\right)
\end{gathered}
$$

where:

$$
\begin{aligned}
V_{t}{ }^{(k)}(i)= & \text { the value function in state } i \text {, period } t \text {, and iteration } \\
& k^{\text {th }}, \\
P_{t}^{a}(i j)= & \text { the probability of going from state } i \text { to state } j \text { under } \\
& \text { the decision } a \\
f_{t}(i, a)= & \text { the immediate reward in state } i \text { under decision } a, \\
A(i, t)= & \text { the set of admissible actions could be taken by the } \\
& \text { agent in state } i \text { and period } t \\
\operatorname{Re}= & \text { expectation of immediate reward, and } \\
\gamma= & \text { discounted rate. }
\end{aligned}
$$

In other words, to find an updated value function, all admissible actions in the respective state and period must be tested based on Equation 8.1 or 8.3 , and the best value is chosen as a new value function. Therefore, we can introduce another terminology called the action-value function, $Q_{t}(i, a)$. This value demonstrates the expected accumulated reward when a decision maker starts from state $i$ and takes action $a$. Using these new values, the formulation of SDP in Equation 8.4 can be rewritten as follows:

$$
\begin{aligned}
V_{t}^{(k)}(i)= & \max _{a \in \mathrm{A}(i, t)} Q_{t}^{(k)}(i, a)=\max _{a \in \mathrm{A}(i, t)}\left(\operatorname{Re}+\gamma \sum_{j=1}^{N} P_{i j} Q_{t+1}{ }^{(k-1)}\left(j, b^{*}\right)\right) \\
& Q_{t+1}{ }^{(k-1)}\left(j, b^{*}\right)=\max _{b \in \mathrm{A}(j, t+1)} Q_{t+1}{ }^{(k-1)}(j, b) \\
& Q_{t}^{(k)}(i, a)=E\left[\operatorname{Re}+\gamma \max _{b \in \mathrm{A}(j, t+1)} Q_{t+1}{ }^{(k-1)}(j, b)\right]
\end{aligned}
$$

where:

$$
E=\text { expectation operator. }
$$

This is the same formulation of the SDP except it is expressed in terms of action-value function. 
Let us examine the algorithm of Robbins-Monro (Robbins \& Monro, 1951) for calculating the average of a sequence of data iteratively. Suppose we have a sequence of data $X_{1}, X_{2}, X_{3}, \ldots X_{n}, X_{n+1}, \ldots X_{m}$. If the average of the first $n$ inputs is denoted by $\bar{X}_{n}$, and the $(n+1)^{\text {th }}$ observation is $X_{n+1}$, the average of the new sequence of data in terms of the current average and the new occurrence is given by:

$$
\bar{X}_{n+1}=\bar{X}_{n}+\frac{1}{n+1}\left(X_{n+1}-\bar{X}_{n}\right)=\frac{\left(\sum_{i=1}^{n+1} X_{i}\right)}{n+1}
$$

Based on this rule, it is simple to find a new formulation as follows:

$$
\begin{gathered}
Q_{t}{ }^{(k)}(i, a)=Q_{t}{ }^{(k-1)}(i, a)+\frac{1}{N O V(i, a)} \times(\mathrm{Re}+ \\
\left.\gamma \max _{b \in \mathrm{A}(j, t+1)} Q_{t+1}{ }^{(k-1)}(j, b)-Q_{t}^{(k-1)}(i, a)\right) \\
Q_{t}{ }^{(k+1)}(i, a)=Q_{t}{ }^{(k-1)}(i, a)+\alpha \times(\mathrm{R} \mathrm{e}+ \\
\left.\gamma \max _{b \in \mathrm{A}(j, t+1)} Q_{t+1}{ }^{(k-1)}(j, b)-Q_{t}^{(k-1)}(i, a)\right)
\end{gathered}
$$

where:

$$
\begin{aligned}
N O V(i, a) & =\text { the number of visits for action } a \text { in state } \mathrm{i}, \text { and } \\
\alpha & =\text { learning parameter. }
\end{aligned}
$$

This is called the Q-Learning method (Watkins, 1989) in which the transition probabilities based on the Markov Chain Process (MCP) in Equation 8.4 are removed. In other words, this is a model-free algorithm in which the transition probabilities are not used for updating the action values.

\subsubsection{Admissible Actions}

In each period, the agent should choose an action among candidate actions, which are called the admissible actions. In other words, admissible actions are a set of all discrete values of the control variable (release) which are 
within the range between minimum and maximum release volumes. One may determine the set of discrete admissible actions as follows:

$$
\begin{gathered}
\text { All possible actions }=\left\{a_{i}^{t} \mid a_{i}^{t}=d_{\min }^{t}+\frac{d_{\max }^{t}-d_{\min }^{t}}{C-1}(i-1)\right. \\
\text { for } i=1 \ldots C,
\end{gathered}
$$

where:

$$
\begin{aligned}
d_{\min }^{t}= & \text { the minimum release from reservoir in period } t, \\
d_{\max }^{t}= & \text { the maximum release from reservoir in period } t, \text { and } \\
C= & \text { maximum number of discrete release volumes in } \\
& \text { each period. }
\end{aligned}
$$

Because different inflows, as a random variable, may be realized in different time periods even if their initial storage volumes are the same, the set of admissible actions also depends on the inflow assumed in the time period under study. Depending upon whether the lower bound of the inflow or its upper bound is taken, two different ways of defining the set of admissible actions associated with any storage volume may be used: (i) optimistic, and (ii) pessimistic.

A set of admissible actions in the optimistic scheme may be determined according to the following four steps:

1. Compute $I_{\max }^{t}$ :

$$
\begin{aligned}
P\left(I^{t} \leq I_{\max }^{t}\right) & =\left(1-\varepsilon_{1}\right) \\
\left.P\left(e v^{t} \leq e v_{\min }^{t}\right)\right) & =\left(\varepsilon_{1}\right)
\end{aligned}
$$

where:

$$
\begin{aligned}
\varepsilon_{1} & =\text { small value close to zero, } \\
I_{\max }^{t} & =\text { the maximum considered inflow in period } t, \\
I^{t} & =\text { the inflow to reservoir in period } t \\
e v^{t} & =\text { the evaporation from reservoir in period } t, \text { and } \\
e v_{\min }^{t} & =\text { the minimum considered evaporation in period } t .
\end{aligned}
$$


For example, assuming a normal distribution for inflow, we may want to find $I_{\max }$ in Equation 8.11 as follows:

$$
\begin{aligned}
& P_{z}\left(I \leq I_{\max }\right)=97.725 \% \\
& P_{z}(Z \leq 2) \quad=97.725 \%
\end{aligned}
$$

where:

$$
\begin{aligned}
P_{z}= & \text { the normal standard distribution with zero mean and } \\
& \quad \text { variance of one } \\
\mu_{I}^{t}= & \text { the mean of inflow in period } t \\
\sigma_{I}^{t}= & \text { the variance of inflow in period } t, \text { and } \\
\mathrm{Z}= & \text { the variable of normal standard. }
\end{aligned}
$$

2. Find all possible actions:

$$
a_{i}^{t}=d_{\min }^{t}+\frac{d_{\max }^{t}-d_{\min }^{t}}{C-1}(i-1) \text { for } i=1 \ldots C
$$

3. Compute all possible values of the next storage volume $s_{i}^{t+1}$ :

$$
s_{i}^{t+1}=s_{j}^{t}+I_{\max }^{t}-e v_{\min }^{t}-a_{i}^{t} \quad \text { for } i=1 \ldots C
$$

4. Find the admissible actions for the current state with respect to storage values computed in Step 3 (those actions are feasible if the related endof-period storage volume in the above equation does not violate the minimum allowable storage volume $s_{\min }^{t+1}$ ):

$$
\text { Admissible actions }\left(d^{t}\right)=\left\{a_{i}^{t} \mid s_{i}^{t+1} \geq s_{\min }^{t+1}\right\} \text { for } i=1 \ldots \mathrm{C}
$$

where:

$$
S_{\min }^{t+1}=\text { the minimum storage of the next period. }
$$

A similar procedure is followed in the pessimistic scheme, except that the lowest inflow is chosen to specify the set of admissible actions for any state of the system. This inflow can be easily determined from the probability distribution function or historical data of the random inflow. 


\subsubsection{Action Policies}

There are four common action policies described in the literature: random, greedy, $\mathcal{E}$-greedy, and Softmax (Sutton \& Barto, 1998; Gosavi, 2003).

In the random policy, there is no action preference. This means that the probabilities of choosing actions are the same and equal $1 /|A(i)|$, where $|A(i)|$ is the number of admissible actions in state $i$.

In the greedy policy, the agent has to pick the best one out of all admissible actions in every iteration with respect to the last estimate of action-value functions for all admissible actions in the respective state. In other words, the agent exploits the current knowledge without considering what may happen in the future. In this strategy, the agent does not try actions with a low immediate reward in the case of maximizing the objective function; therefore, there is no knowledge about what would eventually happen in the future if those actions were chosen.

In the $\mathcal{E}$-greedy policy, the greedy action is chosen for most of the time; however, once in a while, the agent tries to choose an action among all admissible actions with probability of $\varepsilon /|\mathrm{A}(i)|$. In other words, the probability of taking the non-greedy actions is $\varepsilon$. Furthermore, the probability of the greedy action in each iteration is $1-\mathcal{E}+\varepsilon /|\mathrm{A}(i)|$ (Sutton and Barto 1998). It is worth mentioning that when the agent is to take a nongreedy action, because it does not know whether the current greedy action remains greedy in the next iterations, it has to consider all admissible actions as non-greedy actions. Therefore, if the agent wants to take non-greedy action, the current greedy action will have a chance of $\mathcal{\varepsilon} /|\mathrm{A}(i)|$ to be chosen again.

In contradiction to the greedy policy, another strategy can be defined in which the proportion of exploration versus exploitation changes as the process of the learning continues. For example, in an online learning when there is no knowledge about the system under study, an agent intends to do more explorations to discover new situations. However, with achieving more experience, it tries to keep the system stable and avoid taking more risk to change the situation. In other words, the proportion of exploration is usually much higher than exploitation at the starting of the learning process and diminishes after achieving more experience. This type of policy could be conducted by the Softmax policy which is derived from the Gibbs or Boltzmann distribution. In this strategy, the probabilities of choosing actions in every iteration are achieved based on the following formula: 


$$
P(a)=\frac{e^{Q(i, a) / \tau}}{\sum_{b \in|\mathbf{A}(i)|} e^{Q(i, b) / \tau}}
$$

where:

$$
\tau=\text { a positive number called temperature. }
$$

With high temperature, the probabilities of taking all actions are the same, and small temperature causes higher probabilities for actions with large action values. This means that when $\tau$ is very large, the agent explores all actions, and with small $\tau$, it becomes more interested in the greedy actions (exploitation).

\subsubsection{Interpolation}

Although the process of transition from one storage level to another is continuous and controlled by the constraint of the balance equations in reservoir optimization model, there is no guarantee that the current storage or the next storage is exactly one of those discrete values, which are considered for storage levels. However, the process of updating of actionvalue functions is only performed for discrete values.

One easy way to tackle this problem is to find the closest discrete value to the current or next storage level and perform the process of taking action and updating action values based on these values. However, this updating seems to be error-prone because the respective storage only partially belongs to this state. To increase the accuracy, we might use some kind of linear or non-linear interpolation to handle this problem and decrease the simulation error. To interpolate linearly, firstly, we have to find two successive values $s^{-(t)}$ and $s^{+(t)}$ which are the closest discrete values to the current storage. Finally, the degree of closeness of the current storage to these boundaries should be computed using the following formulation:

$$
w_{1}=1-\frac{s^{(t)}-s^{-(t)}}{s^{+(t)}-s^{-(t)}}, \quad w_{2}=1-\frac{s^{+(t)}-s^{(t)}}{s^{+(t)}-s^{-(t)}}
$$

In other words, larger values for $w_{1}$ or $w_{2}$ demonstrate the higher degree of closeness to the pertaining state. For instance, $w_{l}=1$ and $w_{2}=0$ means that the related storage is exactly the same as $s^{-(t)}$. These weights can be 
similarly computed for the next storage by substituting $t$ with $t+1$ in the above formulations. Considering these weights for the next storage, the process of updating action-value function can be performed as follows:

$$
\begin{gathered}
Q_{1, t}{ }^{(k)}(i, a)=Q_{t}{ }^{k-1}(i, a)+\alpha \times(\mathrm{Re}+ \\
\left.\gamma \max _{b \in \mathrm{A}(j, t+1)} Q_{t+1}{ }^{(k-1)}(j, b)-Q_{t}{ }^{k-1}(i, a)\right) \\
Q_{1, t}{ }^{(k)}(i, a)=Q_{t}{ }^{k-1}(i, a)+\alpha \times(\mathrm{Re}+ \\
\left.\gamma \max _{b \in \mathrm{A}(j+1, t+1)} Q_{t+1}{ }^{(k-1)}(j+1, b)-Q_{t}{ }^{k-1}(i, a)\right)
\end{gathered}
$$

where $j$ and $j+1$ are indices for two closest successive discrete values related to the next storage.

The new updated action-value function, therefore, is computed as follows:

$$
Q_{t}{ }^{(k)}(i, a)=w_{1} \times Q_{1, t}(i, a)+w_{2} \times Q_{2, t}(i, a)
$$

\subsection{Results for a Single Reservoir Problem}

\subsubsection{Data Set}

The data for a single reservoir application has been derived from Fletcher (Fletcher, 1995). A year (12 months) is considered as a cycle in this case.

The minimum and maximum storage and release in the different months of a year are given in Table 8.1.

Table 8.1 Boundary condition for storage and release each month

\begin{tabular}{ccccccccccccc}
\hline Month & 1 & 2 & 3 & 4 & 5 & 6 & 7 & 8 & 9 & 10 & 11 & 12 \\
\hline $\begin{array}{c}\text { Max. } \\
\text { Storage }\end{array}$ & 8 & 8 & 8 & 8 & 8 & 8 & 8 & 8 & 8 & 8 & 8 & 8 \\
\hline $\begin{array}{c}\text { Min. } \\
\text { Storage }\end{array}$ & 1 & 1 & 1 & 1 & 1 & 1 & 1 & 1 & 1 & 1 & 1 & 1 \\
\hline $\begin{array}{c}\text { Max. } \\
\text { release }\end{array}$ & 4.0 & 4.0 & 6.0 & 6.0 & 7.5 & 12.0 & 8.5 & 8.5 & 6.0 & 5.0 & 4.0 & 4.0 \\
\hline $\begin{array}{c}\text { Min. } \\
\text { release }\end{array}$ & 0.0 & 0.0 & 0.0 & 0.0 & 0.0 & 0.0 & 0.0 & 0.0 & 0.0 & 0.0 & 0.0 & 0.0 \\
\hline
\end{tabular}


Inflow to the reservoir is normally distributed with the monthly averages given in Table 8.2. The coefficient of variance $\left(\sigma_{I^{t}} / \mu_{I^{t}}\right)$ in each month of a year is a pre-determined value used to obtain the variance of inflow. Moreover, the evaporation from the reservoir is neglected.

Table 8.2 The average inflow in each month of a year.

\begin{tabular}{ccccccccccccc}
\hline Month & 1 & 2 & 3 & 4 & 5 & 6 & 7 & 8 & 9 & 10 & 11 & 12 \\
\hline $\begin{array}{c}\text { Average } \\
\text { inflow }\end{array}$ & 3.4 & 3.7 & 5.0 & 5.0 & 7.0 & 6.5 & 6.0 & 5.5 & 4.3 & 4.2 & 4.0 & 3.7 \\
\hline
\end{tabular}

The objective function in the Fletcher's model is to maximize the power generation. The benefit of release per unit is approximated for each month in Table 8.3.

Table 8.3 The benefit of release per unit for each month of a year.

\begin{tabular}{ccccccccccccc}
\hline Month & 1 & 2 & 3 & 4 & 5 & 6 & 7 & 8 & 9 & 10 & 11 & 12 \\
\hline $\begin{array}{c}\text { Benefit of } \\
\text { release }\end{array}$ & 1.4 & 1.1 & 1.0 & 1.0 & 1.2 & 1.8 & 2.5 & 2.2 & 2.0 & 1.8 & 2.2 & 1.8 \\
\hline
\end{tabular}

Each state and decision variable was discretized into 55 and 7 equal intervals, respectively. Therefore, there are eight discrete values for storage level and 56 possible actions that agent may take. Moreover, the storage level as a state of the system after each interaction with the environment should belong to one of discrete values of the storage. Furthermore, as previously mentioned, the action that the agent takes can lead to a release, which is completely different from the action taken; however, the process of updating action-value functions is performed based on this action.

In Q-Learning, each episode has 30 years, which is repeated 1000 times for one complete experiment. Further more, the Q-Learning formulation used in these experiments is derived from the value iteration version of SDP (Gosavi, 2003) with discounted rate of $\gamma=0.9$.

The final decisions of the Q-Learning algorithm are compared to the optimal decisions derived from the SDP to verify the performance of the learning process. As it is clear, in spite of having some kind of estimation 
errors in SDP, the performance measures such as average and variance of gain resulted from simulation are expected to be the best values in this case study. Therefore, to demonstrate the high efficiency of Q-Learning, we have to achieve the same or very close performance as SDP. It is worth mentioning that since the transition from a state (storage) to another based on the balance equations is continuous and there is no discretization for random variables such as inflow and evaporation in Q-Learning, the error of discretization in the learning method is definitely less than SDP.

To find the efficiency of the Q-Learning or SDP, a simulation for 10000 years is implemented. There is also no spillage loss in the system. Different investigations were implemented and are described in the next section.

\subsubsection{Effect of Action Policies in Pessimistic and Optimistic Schemes}

Tables 8.4-8.6 show performance measures of Q-Learning and SDP resulting from simulation for pessimistic and optimistic schemes of admissible actions with consideration of a constant or a variable learning parameter. In order to find the admissible actions in these schemes for every month of a year in the experiments, the minimum and maximum inflows are computed based on Equations 8.11 and 8.13 as follows:

$$
\begin{aligned}
& I_{\text {max }}^{t}=\mu_{I}{ }^{t}+2 \sigma_{I}^{t}, \\
& I_{\text {min }}^{t}=\mu_{I}^{t}-2 \sigma_{I}^{t} .
\end{aligned}
$$

Table 8.4 The performance of the value-iteration version of the discounted SDP method for the pessimistic and optimistic view of maximizing benefit $(\gamma=0.9)$.

\begin{tabular}{cccccc}
\hline View & $\begin{array}{c}\text { Mean } \\
\text { (yearly) }\end{array}$ & $\begin{array}{c}\text { Variance } \\
\text { (yearly) }\end{array}$ & $\begin{array}{c}C V \\
\text { of benefits } \\
\text { (yearly) }\end{array}$ & Failure & $\begin{array}{c}\text { Optimization } \\
\text { time (sec) }\end{array}$ \\
\hline Pessimistic & 97.7912 & 58.2516 & 0.078 & $9.57 \%$ & 50.3194 \\
\hline Optimistic & 102.21 & 75.61 & 0.0851 & $46.1 \%$ & 51.06 \\
\hline
\end{tabular}

$C V=\sigma / \mu$ 
Table 8.5: The performances of Q-Learning based on the value-iteration version of the discounted method in the pessimistic view for maximizing benefit $(\gamma=0.9 . \quad C V=\sigma / \mu)$.

\begin{tabular}{ccccccc}
\hline Policy & $\begin{array}{c}\text { Learning } \\
\text { parameter }\end{array}$ & $\begin{array}{c}\text { Mean } \\
\text { (yearly) }\end{array}$ & $\begin{array}{c}\text { Variance } \\
\text { (yearly) }\end{array}$ & $\begin{array}{c}C V \\
\text { of benefits } \\
\text { (yearly) }\end{array}$ & Failure & $\begin{array}{c}\text { Learning } \\
\text { time } \\
\text { (sec) }\end{array}$ \\
\hline \multirow{2}{*}{ Random } & $\alpha=0.5$ & 96.98 & 66.77 & 0.0843 & $9.2 \%$ & 149 \\
& $A=0.5$ & 95.62 & 53.18 & 0.076 & $20 \%$ & 98 \\
\hline \multirow{2}{*}{ Softmax } & $\alpha=0.5$ & 96.68 & 69.63 & 0.078 & $9.3 \%$ & 649 \\
& $A=0.5$ & 96.61 & 57.01 & 0.085 & $17 \%$ & 200 \\
\hline \multirow{2}{*}{ Greedy } & $\alpha=0.5$ & 63.85 & 29.54 & 0.1079 & $76.3 \%$ & 187 \\
& $A=0.5$ & 62.4 & 45.3 & 0.0667 & $74 \%$ & 137 \\
\hline \multirow{2}{*}{$\varepsilon$-greedy } & $\alpha=0.5$ & 95.57 & 50.16 & 0.0741 & $19.4 \%$ & 95 \\
\hline
\end{tabular}

Table 8.6 The performance of Q-Learning based on the value-iteration version of the discounted method in the optimistic view of maximizing benefit $(C V=\sigma / \mu)$.

\begin{tabular}{ccccccc}
\hline Policy & $\begin{array}{c}\text { Learning } \\
\text { parameter }\end{array}$ & $\begin{array}{c}\text { Mean } \\
\text { (yearly) }\end{array}$ & $\begin{array}{c}\text { Variance } \\
\text { (yearly) }\end{array}$ & $\begin{array}{c}C V \\
\text { of benefits } \\
((\text { yearly })\end{array}$ & Failure & $\begin{array}{c}\text { Learning } \\
\text { time } \\
(\mathrm{sec})\end{array}$ \\
\hline \multirow{2}{*}{ Random } & $\alpha=0.5$ & 98.06 & 75.99 & 0.0889 & $22.59 \%$ & 123 \\
\hline \multirow{2}{*}{ Softmax } & $\alpha=0.5$ & 97.47 & 74.47 & 0.0885 & $35.99 \%$ & 95 \\
\hline \multirow{2}{*}{ Greedy } & $\alpha=0.5$ & 97.69 & 79.79 & 0.0914 & $26.57 \%$ & 385 \\
\hline \multirow{2}{*}{\begin{tabular}{c}
$*$ \\
\multirow{2}{*}{-greedy }
\end{tabular}} & $\alpha=0.5$ & 71.03 & 44.84 & 0.0943 & $61.98 \%$ & 160 \\
\hline
\end{tabular}

The last column in Table 8.4 is pertinent to the time of optimization in SDP; however, in Table 8.5 and 8.6 it is related to the time that the learning process needs to find the final policy through the Q-Learning algorithm. 
Moreover, the failure column in these tables represents the total percentage of time in which the maximum and minimum storages are violated.

Figures 8.2-8.5 compare the final decision of SDP and Q-Learning in the months of January and June for pessimistic and optimistic views.

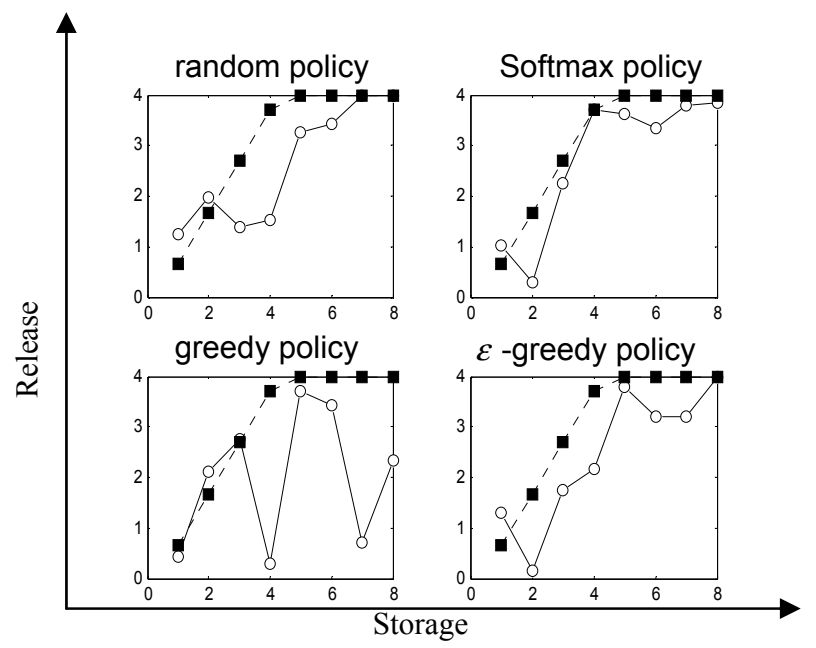

Figure 8.2 Comparison between optimal decisions in SDP and QLearning in the month of January for different policies in the pessimistic view $(\gamma=0.9 \& \alpha=0.5)$. Solid line: Q-learning, dashed line: SDP.

Based on conducted experiments, the results can be summarized:

1. The greedy policy is not a suitable policy for the agent in order to take actions in terms of the gain average. Tables 8.5 and 8.6 show that the average of gain in optimistic and pessimistic views for the greedy policy are the lowest values among other policies. For example, the averages of gain for constant learning rate are 63.85 and 71.03 in pessimistic and optimistic, respectively; however, the best averages are related to the $\mathcal{E}$-greedy policy with 95.57 and 98.86 under the same conditions. This is also demonstrated in Figure 8.2 and 8.3 for the pessimistic view when all optimal actions in different policies follow the same actions derived from SDP except the greedy policy in which some actions vary significantly from the SDP actions in different storage levels. 


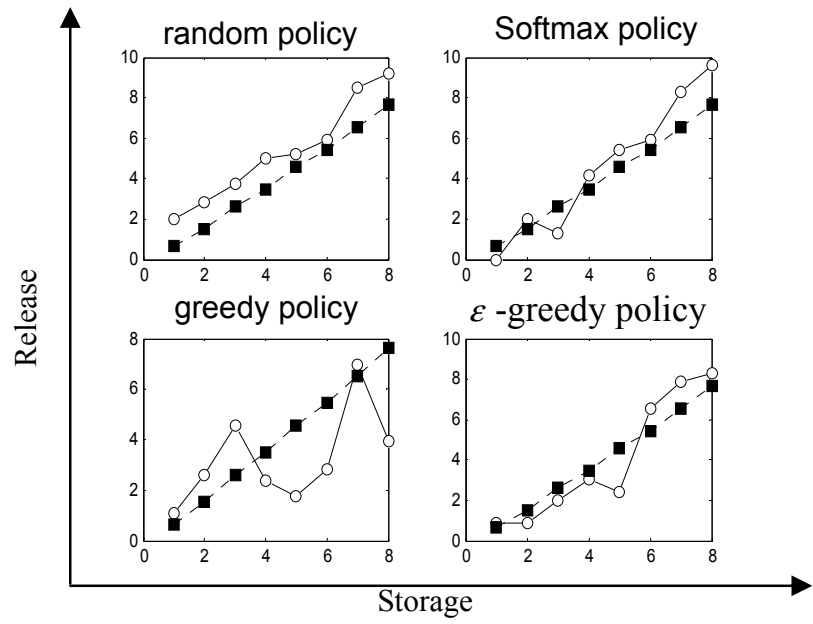

Figure 8.3 Comparison between optimal decisions in SDP and QLearning in the month of June for different policies in the pessimistic view $(\gamma=0.9 \& \alpha=0.5)$. Solid line: $\mathrm{Q}$-learning, dashed line: SDP.

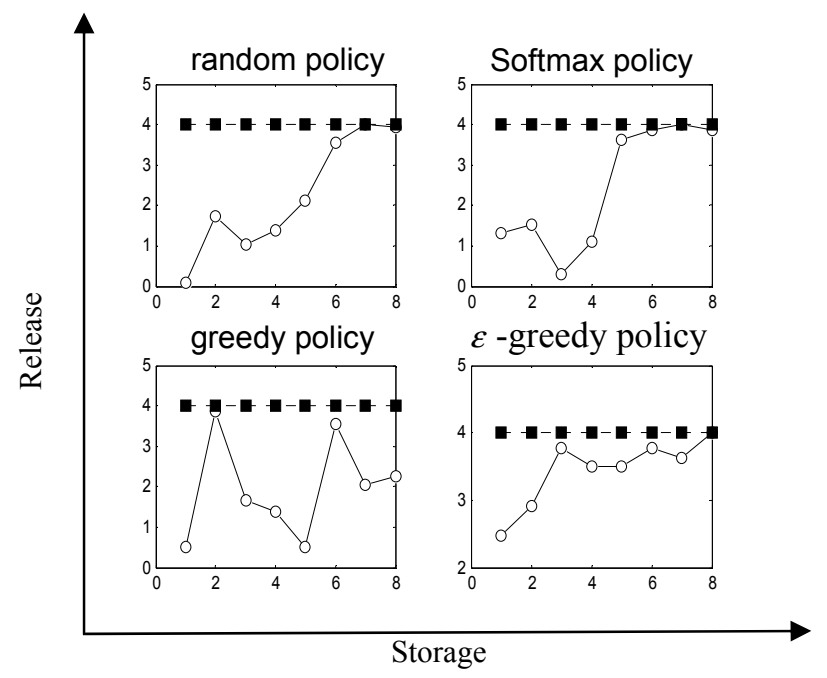

Figure 8.4 Comparison between optimal decisions in SDP and the QLearning in month of January for different policies in the optimistic view $(\gamma=0.9 \& \alpha=0.5)$. Solid line: Q-learning, dashed line: SDP. 


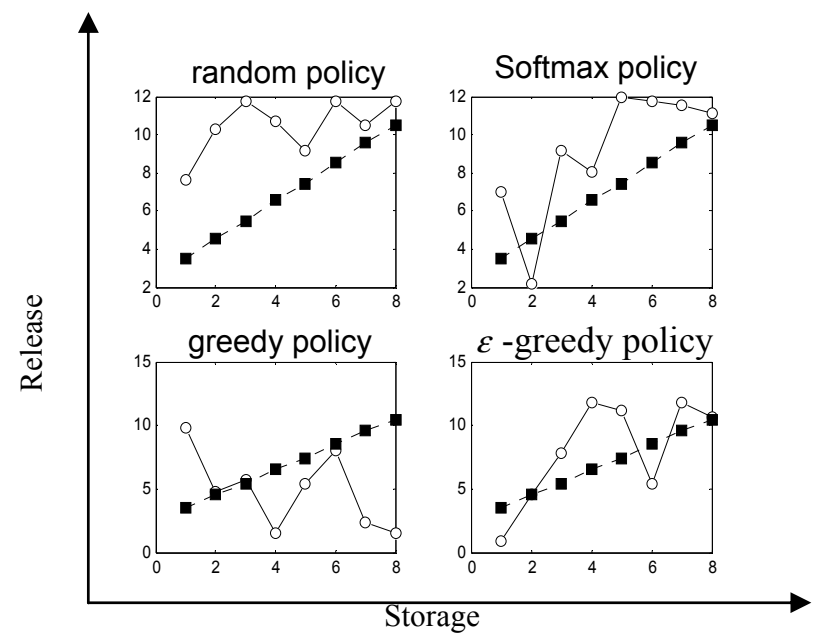

Figure 8.5 Comparison between optimal decisions in SDP and the QLearning in the month of June for different policies in the optimistic view $(\gamma=0.9 \& \alpha=0.5)$. Solid line: Q-learning, dashed line: SDP.

2. Based on what it is illustrated in Tables 8.5 and 8.6 and verified in Figures 8.2 and 8.3, there is no significant difference between the random, Softmax, and $\varepsilon$-greedy policies in terms of average of benefit and the best actions. However, the variance of $\varepsilon$-greedy policy is minimum compared to other policies and even less than the SDP in the pessimistic and optimistic views. Moreover, the uncertainty $(\mathrm{CV})$ in the $\varepsilon$-greedy policy is significantly lower than the SDP in both views and the same effect happens for the percentage of failure in the optimistic view (18.71\% for $\varepsilon$-greedy policy with a constant learning parameter against $46.1 \%$ for SDP).

3. As expected and observed in the Tables 8.5 and 8.6, the optimal actions based on the optimistic view have a better chance to achieve more benefit because of action diversity in different states compared to the pessimistic view. However, the annual risk (annual variance) and uncertainty $(\mathrm{CV})$ of this policy does not increase for $\varepsilon$-greedy policy in the optimistic view (e.g. 50.16 in the pessimistic against 
48.63 in the optimistic view for a constant learning parameter). In other words, the annual risk and uncertainty for this policy in the optimistic view are almost the same with the pessimistic view, which means that we achieve more benefit without increasing the risk and stochasticity in the objective function. Moreover, the percentage of failure in the respective policy in the optimistic view is almost the same as in pessimistic view $(19.4 \%$ in the pessimistic against $18.71 \%$ in the optimistic). Therefore, it seems that the optimistic view might be a better option to be chosen in real-world applications.

4. As observed in Figures 8.4 and 8.5, the optimal actions extracted by the Q-Learning based on random, Softmax, and $\mathcal{E}$-greedy policies do not follow the actions derived by SDP in lower storage levels; however, they are almost the same in higher volumes of storage. It seems that this is related to input data because the agent experiences higher storage levels during the simulation much more than lower levels (e.g., the agent visits the storage level 1 in month 1 only 175 times in the optimistic view while it experiences level 88015 times in the same month). This could be the main reason that action-value functions of these stateaction pairs are updated only a few times; therefore, actions related to these states might not be optimal or near-optimal actions. As previously mentioned, to find the optimal action-value functions, the agent should visit all possible action-state pairs as frequently as possible. Moreover, because the Q-Learning method updates the action-value functions asynchronously, and because the agent visits more the storages with high level during the simulation, this could increase the error.

5. As it is represented in Tables 8.5 and 8.6, the performance of the learning process is almost the same for constant and variable learning parameters for all policies. 


\subsection{Conclusions}

Based on the preliminary results, it becomes clear that the RL techniques can obtain near optimal solutions with high robustness for a single reservoir application. It has also been shown that in the respective case study, the QLearning algorithm achieved high performance in terms of variance, coefficient of variance, and percentage of failure compared to SDP method. This technique could be a promising method to be employed in the management of multi-reservoir applications in the future.

\section{References}

Fletcher S. G. 1995, A new formulation for the stochastic control programming optimization to a Northern Ontario Hydro Power System. Ph.D. Dissertation, Dept. of systems design engineering, University of Waterloo, Canada.

Gosavi A. 2003, Simulation-based optimization: parametric optimization techniques and reinforcement learning. Kluwer academic publisher, Norwel Massachusetts.

Hall W., Harboe R., Yeh W., and Askew A. 1969, Optimum fire power output from a two reservoir system by incremental dynamic programming. Contribution \# 130. Water Research Center, Univ. of California, Los Angeles.

Labadie J. 2004, Optimal operation of multireservoir systems: State-of-the-art review. J. Water Resou. Plan. And Manage, ASCE, 130(2), 93-111.

Larson R. 1968, State increment dynamic programming. Elsevier, New York.

Robbins H., and Monro, S. 1951, A stochastic approximation method. Ann. Math. Statist, $22,400-407$.

Nopmongcol P., and Askew A. 1976, Multilevel Incremental dynamic programming. Water Resour. Res., 12(6), 1291-1297.

Ponnambalam K. 1987, Optimization of the integrated operation of multi-reservoir irrigation systems. Ph.D. Dissertation, Department of Civil Engineering, University of Toronto, Toronto, Canada.

Ponnambalam K., and Adams Barry J. 1996, Stochastic optimization of multireservoir systems using a heuristic algorithm: case study from India. Water Resour. Res., 32(3), 733-742.

Sutton R. S., and Barto A. G. 1998, Reinforcement Learning. The MIT press, Cambridge, Massachusetts.

Turgeon A. 1981, A decomposition method for the long term scheduling of reservoirs in series. Water Resour. Res., 17, 1565-1570.

Watkins C.G.H., and Dayan P. 1992, Q-Learning. Technical Report, Machine Learning, 8, 279-292.

Watkins C.G.H. 1989, Learning from delayed rewards. PhD Thesis, University of Cambridge. 
